\title{
Research on Indoor Positioning Algorithm of Single Reader Based on Gated Recurrent Unit
}

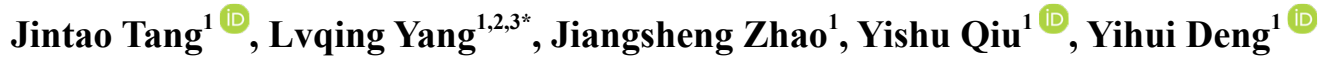 \\ ${ }^{1}$ School of Informatics, Xiamen University, Xiamen 361005, China \\ ${ }^{2}$ State Key Laboratory of Process Automation in Mining, Beijing 102600, China \\ ${ }^{3}$ Beijing Key Laboratory of Process Automation in Mining \& Metallurgy, Beijing 102600, China \\ Email: lqyang@xmu.edu.cn
}

Received: 25 September 2020; Revised: 4 December 2020; Accepted: 26 December 2020

\begin{abstract}
With the development of the Internet of Things and Radio Frequency Identification (RFID), indoor positioning technology as an important part of positioning technology, has been attracting much attention in recent years. In order to solve the problems of low precision, high cost and signal collision between readers, a new indoor positioning algorithm based on a single RFID reader combined with a Double-order Gated Recurrent Unit (GRU) are proposed in this paper. Firstly, the reader is moved along the specified direction to collect the sequential tag data. Then, the tag's coordinate is taken as the target value to train models and compare them with existing algorithms. Finally, the best Gated Recurrent Unit positioning model is used to estimate the position of the tags. Experiment results show that the proposed algorithm can effectively improve positioning accuracy, reduce the number of readers, cut down the cost and eliminate the collisions of reader signals.
\end{abstract}

Keywords: indoor positioning, GRU, RFID, signal collision

\section{Introduction}

Due to the signal shielding and weakening of buildings, the Global Navigation Satellite System (GNSS) and other positioning technologies can't be applied indoors. Wireless indoor positioning technologies are constantly developing and have good application scenarios, such as underground garages, museums, mines, etc. [1] The main positioning technologies used in the contemporary industry include WiFi positioning, Bluetooth positioning, RFID positioning, Infrared laser positioning, Ultrasonic positioning and Ultra Wideband (UWB) positioning [2-7]. As can be seen from Table 1, RFID has the characteristics of fast scanning, anti-pollution ability and strong durability in contrast. However, collision, penetration, interference and other factors will have a great impact on the accuracy of positioning. Traditional indoor positioning algorithms such as LANDMARC, VIrtual Reference Elimination (VIRE), Triangular Centroid method, Time of Arrival (TOA)/ Angle of Arrival (AOA) hybrid parameter positioning method use geometric methods for positioning, and the accuracy is above 1 meter, which does not meet the accuracy requirements of indoor positioning research [8-12]. Therefore, in recent years, some researchers put forward the application of artificial intelligence technology in indoor positioning algorithms to improve the accuracy of positioning. However, the common practice is to place multiple readers in different parts of the room to read the signal characteristics of the positioning target, which will not only increase the cost but also introduce the factor of the signal collision between readers. Therefore, this

Copyright (C2021 Lvqing Yang, et al.

DOI: https://doi.org/10.37256/aie.212021663

This is an open-access article distributed under a CC BY license

(Creative Commons Attribution 4.0 International License)

https://creativecommons.org/licenses/by/4.0/ 
paper proposes the indoor positioning algorithm combining GRU with a single moving reader, which is a very specific positioning problem: a single reader moves along a predefined path and at the end of the path it identifies the position of RFID tags, which can fundamentally eliminate the signal collision between readers, reduce the cost, and improve the positioning accuracy. The advantage is that only one reader is used, which can fundamentally eliminate signal collisions between readers and reduce costs. At the same time, deep learning algorithms are introduced for data mining, which will improve positioning accuracy.

Table 1. Comparison table of positioning techniques

\begin{tabular}{ccccc}
\hline Techniques & Cost & Precision & Advantages & Disadvantage \\
\hline WiFi & low & $3-20 \mathrm{~m}$ & Extensive network & Unstable, Positioning drift \\
Bluetooth & low & $1-8 \mathrm{~m}$ & Small size, Easily integrate and popularize & Short propagation distance \\
RFID & middle & $1.0-1.9 \mathrm{~m}$ & Fast scanning, anti-pollution ability and durability & Low security \\
Infrared layser & high & $<0.5 \mathrm{~m}$ & High precision & Straight line of sight \\
Ultrasonic & high & $<20 \mathrm{~cm}$ & High precision & Environmental interference \\
UWB & high & $<10 \mathrm{~cm}$ & High precision, strong penetrating ability & High cost and high difficulty in research \\
\hline
\end{tabular}

\section{Related work}

At present, artificial intelligence-related algorithms were gradually maturing, and the combination of machine learning, deep learning and RFID positioning technology has gradually become a research hotspot. Yun Deng, Yan Zhu et al. (2019) proposed an indoor positioning algorithm combining k-means, Support Vector Machine (SVM) and Backpropagation (BP) neural network, and its mean square error of positioning was less than $1 \mathrm{~m}$ [13]. However, multimodel fusion was prone to overfitting, which reduced the generalization of the model. Yinlong Zhao, Shengbiao An et al. (2018) proposed to use SVM for interval classification in the first stage, and establish a separate regression model in the classification subspace in the second stage, thereby reducing the burden of establishing Support Vector Regression (SVR) models and increasing the accuracy of WiFi indoor positioning to $1.5 \mathrm{~m}$ [14]. But the instability of the WiFi signal itself makes the final positioning accuracy still unable to reach the decimeter level. Ningjia Song, Yinghua Cui et al. (2018) proposed an RFID indoor positioning algorithm that combines Generalized Regression Neural Network (GRNN) and uses a genetic algorithm to adjust hyper-parameters, which has high positioning accuracy even with a small number of samples [15]. However, it is necessary to calculate the Guass function value between the test samples and all training samples at the mode layer, which will bring huge time loss in a big data environment. In addition, most of the current indoor positioning based on artificial intelligence algorithms use multiple readers or base stations to obtain multi-dimensional features of the positioning target for training, which will lead to high costs. Therefore, how to obtain higher positioning accuracy at a low cost is a problem that needs to be solved at present. In contrast, the advantage of the positioning algorithm proposed in this paper is that only one reader is used, which greatly reduces the positioning cost. Moreover, we use GRU to analyze the time sequence of collected signals and discover high-order physical signs to improve the accuracy of positioning.

\section{Methods}

\subsection{Free space signal attenuation model}

As the loss of electromagnetic signals in the air, Received Signal Strength Indication (RSSI) has a logarithmic relationship with distance, which can be used in this paper to construct the signal timing characteristics of the positioning target. In order to more truly restore the influence of the indoor complex environment on signal propagation, this paper adopts the free space signal attenuation model with random variables to collect the simulation experiment 
data (detailed improvement methods are introduced in section 4.1):

$$
R S S I(d)=R S S I\left(d_{0}\right)-10 n \lg \left(\frac{d}{d_{0}}\right)+X_{\sigma}
$$

In formula (1), $d_{0}$ is the reference distance between the selected RFID tag and reader; $\operatorname{RSSI}\left(d_{0}\right)$ is the base signal loss; $n$ is the path loss coefficient; $d$ is the distance between the target to be tested and the reader; $X_{\sigma}$ is used to simulate indoor noise, which is normally distributed.

\subsection{RNN evolution and indoor location}

The indoor positioning problem solved in this paper is a time-series regression problem, which can be solved by the Recurrent Neural Network (RNN) family models. As shown in Figure 1, the RSSI sequence of the positioned tag collected by the mobile reader is used as the feature input, and the tag's coordinates are used as the target output to train the model and finally, use the trained model to predict the coordinates of the target.

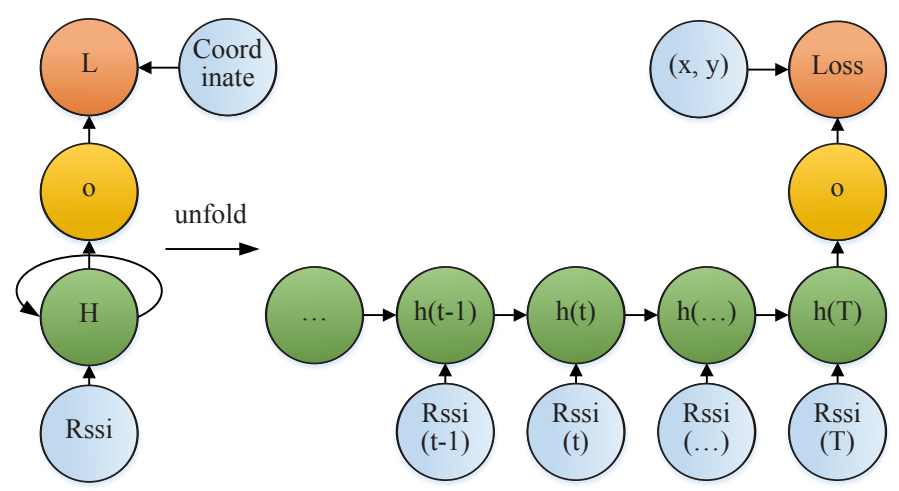

Figure 1. RNN positioning model

As shown in Figure 2, RNN is the simplest recurrent neural network structure. The output of each time step is not only related to the input of the current time, but also related to the output of the previous time step. The hidden unit shares parameters at each time step, and uses the BPTT (Back-propagation Trough Time) algorithm to optimize the parameters. However, as the length of the time series increases, RNNs are prone to gradient disappearance or gradient explosion, resulting in long-term dependence problems [16]. Therefore, the gate mechanism proposed by Long-Short Term Memory (LSTM) can effectively decide to forget or retain relevant information, and achieve a better memory effect [17].
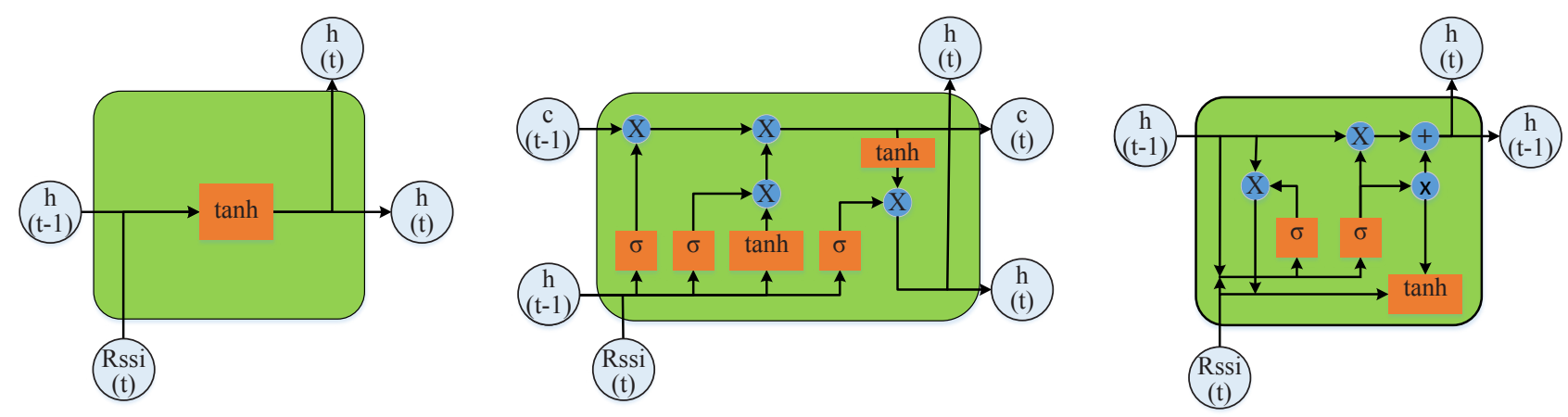

Figure 2. Structure diagram of RNN, LSTM and GRU hidden units 
Different from the gate mechanism of LSTM's input gate, forgetting gate and output gate, the GRU used in this paper only contains reset gate and update gate, so it contains fewer parameters which is better to avoid the overfitting problem when applied to small-scale data [18]. The forward propagation of GRU is shown in Formula (2) to Formula (5). Formula (2) shows the reset gate mechanism of GRU, which helps to capture the short-term and medium-term dependency of timing data. Formula (3) shows the update gate mechanism of GRU, which helps to capture the longterm dependency on timing data.

$$
\begin{gathered}
r_{t}=\operatorname{sigmoid}\left(W^{r} x_{t}+U^{r} h_{t-1}\right) \\
z_{t}=\operatorname{sigmoid}\left(W^{z} x_{t}+U^{z} h_{t-1}\right) \\
\widetilde{h_{t}}=\tanh \left(W x_{t}+r_{t} \odot U h_{t-1}\right) \\
h_{t}=z_{t} \odot h_{t-1}+\left(1-z_{t}\right) \widetilde{h}_{t}
\end{gathered}
$$

In this paper, each $x_{t}$ is a one-dimensional vector, and only saves the tag signal strength collected by the RFID reader at the time of t. Finally, the fully connected output is a two-dimensional vector, which is the predicted label coordinates. Therefore, the parameters of the RNN, LSTM, and GRU positioning models mentioned in this article can be described as formula (6):

$$
\text { Total Params }=k \times[T \times(T+l x)+T]+T \times l y+l y
$$

In formula (6), the $k$ values of RNN, LSTM, and GRU are 1, 4, 3 respectively. $T$ represents the time steps of the recurrent network, $l x$ represents the length of the $x$ vector, and $l y$ represents the length of the $y$ vector. In this paper, $T=$ $100, l x=1, l y=1$. Therefore, the parameters of RNN, LSTM, and GRU are 10402, 41002, and 30802 respectively.

\subsection{Double-order GRU indoor positioning algorithm}

The first-order difference refers to the difference between two adjacent items in the discrete function. As very important feature information in time series analysis, it can effectively reflect the relationship between frames and reduce the volatility of data, thereby making the sequence more stable. Therefore, in order to further improve the positioning accuracy, this paper proposes a Double-order GRU(D-GRU) indoor positioning algorithm that adds the firstorder difference information of the data.

Figure 3 shows the structure of the D-GRU positioning model. It mainly consists of three parts:

(1) The original GRU part: take $R_{-} V e c$ collected by the moving reader as input, and output $H(T)$ through GRU:

$$
R_{-} V e c=\left[R s s i_{1}, R_{s s i}, \cdots, R s s i_{T}\right]
$$

(2) The First-order GRU part: take the first-order difference vector of $R_{-} V e c, \Delta R \_V e c$ as input, and output $\Delta H(T)$ through GRU:

$$
\begin{gathered}
\Delta R \_V e c=\left[\Delta R s s i_{1}, \Delta R s s i_{2}, \cdots, \Delta R s s i_{T}\right] \\
\Delta R s s i_{i}=\left\{\begin{array}{c}
R s s i_{i}, i=1 \\
R s s i_{i}-R s s i_{i-1}, i>1
\end{array}\right.
\end{gathered}
$$

(3) Fully connected layer after concatenating: use the tag's coordinates as the target value, and use Mean Square Error (MSE) as the loss function to backpropagate and optimize parameters: 


$$
M S E=\frac{1}{m} \sum_{i=1}^{m}\left(y_{i}-\hat{y}_{i}\right)^{2}
$$

The advantage of such a design is that under the premise that the original-order information is not lost, the firstorder timing information is added to widen the model, so that the model can learn more information, thereby further improving the positioning accuracy.

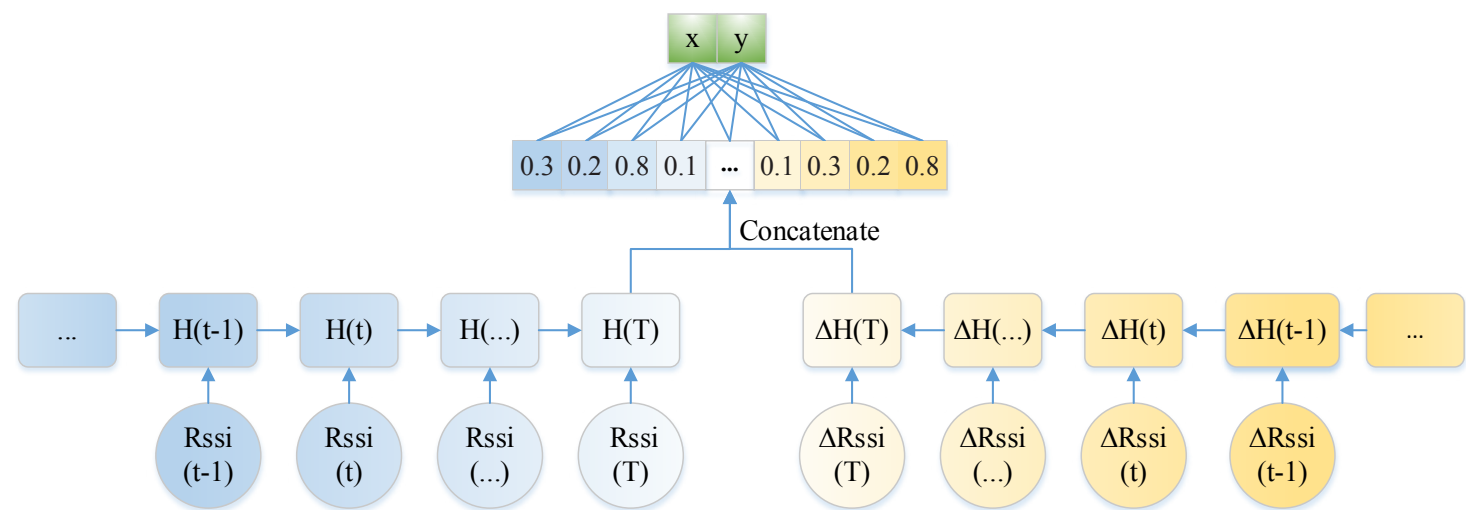

Figure 3. Double-order GRU positioning model

The D-GRU model proposed in this paper merges the output of two independent GRUs, and then outputs the predicted coordinates through a fully connected layer. Therefore, the parameter quantity formula is as follows:

$$
\text { Total Params }=2 \times k \times[T \times(T+l x)+T]+2 \times T \times l y+l y
$$

In formula (11), $k=2, T=100, l x=1, l y=2$, and the meaning is consistent with formula (6), so Total Params $=$ 61602 .

\section{Experiment and simulation}

\subsection{Simulation experiment process}

Our experiment will be carried out in a $10 \mathrm{~m} \times 10 \mathrm{~m}$ simulated indoor environment. As shown in Figure 6, the reader will move along a predefined path to collect the RSSI timing information of the tag, the length is fixed at 100, and the tag coordinate (xi, yi) is recorded together as a piece of data. In order to carry out more realistic reduction experiments and effectively simulate the impact of multipath effects. This paper made the following improvements based on the free space signal attenuation model:

(1) Set $d_{0}$ in Formula (1) to $1.00 \mathrm{~m}$, and use the RFID device shown in Figure 4 to measure $\operatorname{RSSI}\left(d_{0}\right)$;

(2) In the process of generating the distance between the tag and the reader, the RFID ranging error of $-1 \mathrm{~m}$ to $1 \mathrm{~m}$ is added which is larger than the experimental ranging error of many researchers, which can better ensure the credibility of the experimental noise;

(3) Increase the variance of the noise in Formula 1.

After the measurement, the free space signal attenuation model parameters are set as:

$$
d_{0}=1.00 m, \operatorname{RSSI}\left(d_{0}\right)=-18.0, n=2, X_{\sigma} \sim N(0,2)
$$




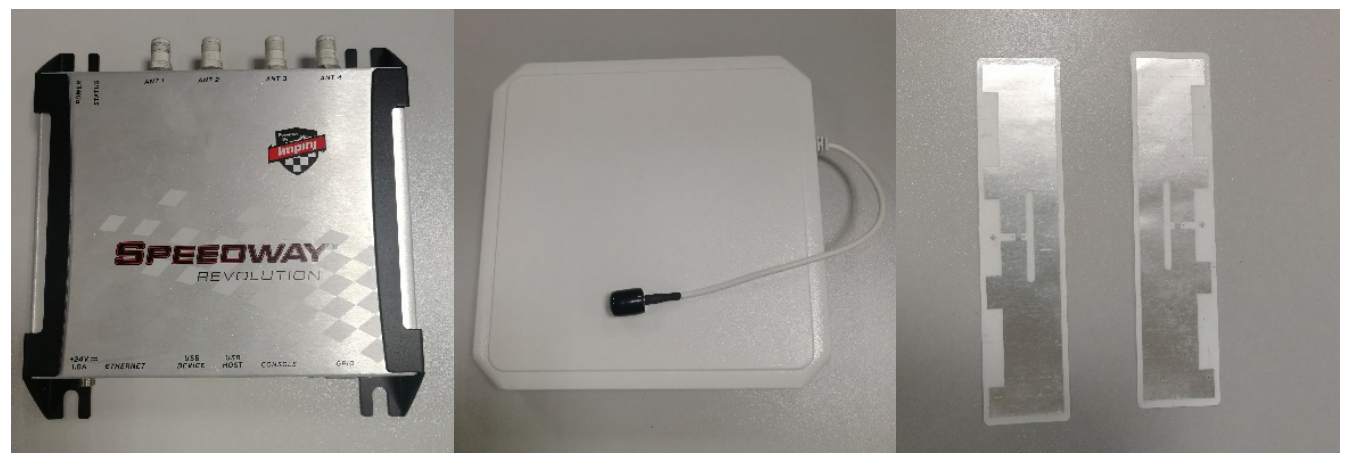

Figure 4. RFID equipment, from left to right are RFID reader, RFID antenna and RFID tag

Data description: Each piece of data is composed of RSSI sequence and coordinates, such as [rssi1, rssi2, ... , rssi100, x, y]. A total of 2000 pieces of data are collected (400 per tag), 80\% of which are used as the training set and $20 \%$ as the test set. Figure 5 shows the random five pieces RSSI sequence line chart, the tags' coordinates are $(6.2,0.8)$, $(1.75,3.83),(7.68,1.81),(9.46,8.68)$ and $(4.74,3.28)$. It can be found in Figure 5:

(1) If the abscissa and ordinate of the tag are relatively small, its line will be at a higher position, otherwise, it will be at a lower position. For example, the position of the yellow polyline corresponding to $(1.75,3.83)$ is the highest intuitively, and the position of the red polyline corresponding to $(9.46,8.68)$ is the lowest intuitively.

(2) The ordinate of the tag is close to the highest point of the polyline. For example, the highest point of (6.2, $0.8)$ and $(7.68,1.81)$ are between 0 and 20 , while the highest points $(1.75,3.83)$ is between 20 and 40 . This is because in an ideal indoor environment with no noise effect, when the reader moves to the point where the line with the tag is perpendicular to the wall, the distance between the tag and the reader is the closest and RSSI is the largest. However, due to the influence of noise and the uneven position of the data collected by the reader, the deviation occurs.

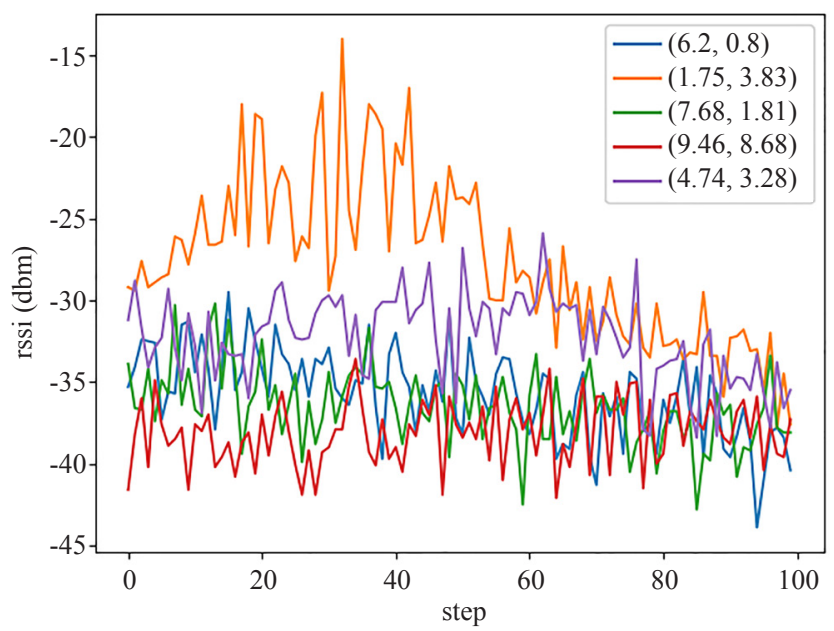

Figure 5. RSSI timing diagram

\subsection{Analysis of experimental results}

In this paper, one of the main reasons for adding the first-order difference information of the time series is because of its stationarity. The left side of Figure 6 shows the RSSI sequence line graph corresponding to the tag at (1.75, 3.83), and the right side of Figure 6 is the result of the corresponding first-order difference sequence calculated by the 
experiment. It can be seen from the experimental results that the first-order difference data oscillates around 0, while the original-order data are in a rising first and then falling trends. In contrast, the first-order difference sequence is more stable.
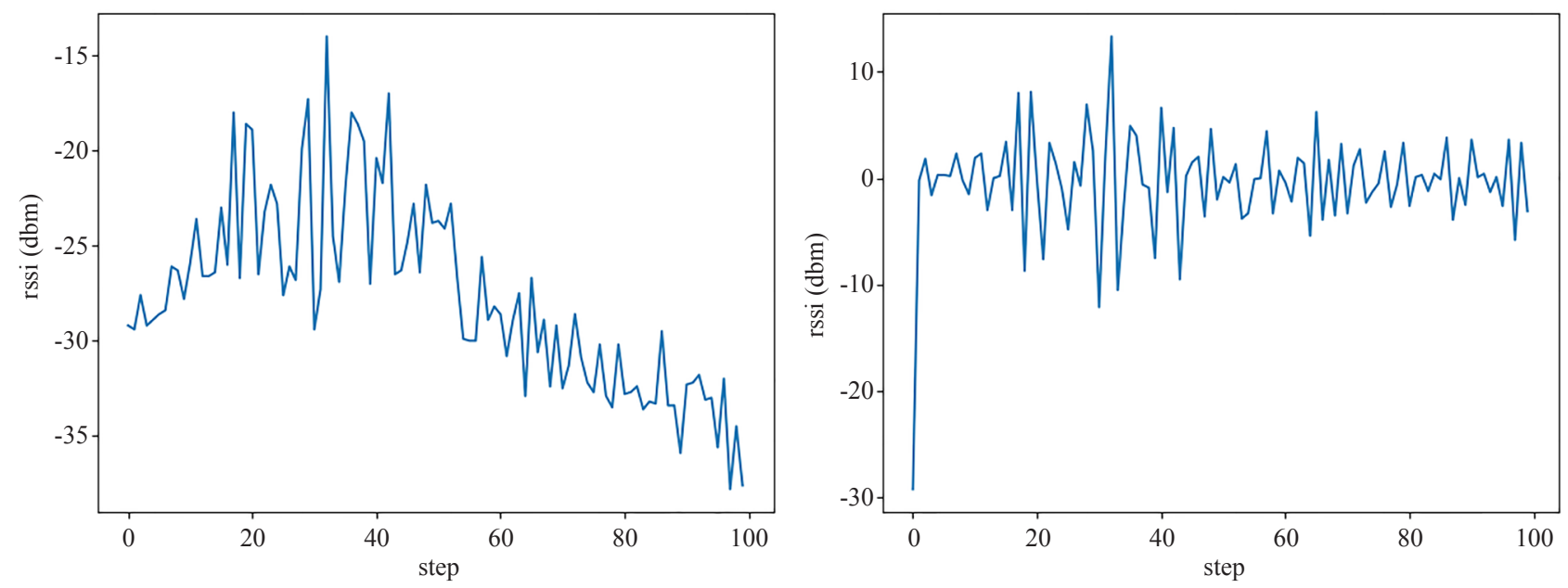

Figure 6. Double-order RSSI timing diagram, original order time sequence diagram on the left and first order time sequence diagram on the right

The task of this paper is to mine the coordinate position of the tag from the messy RSSI time series data. The experiment first uses RNN family models which are good at handling timing problems, including RNN, LSTM and GRU, for training and prediction; Then we use the proposed D-GRU for data mining; finally, Deep Neural Networks (DNN), SVR and GRNN mentioned in the related work section are used as baselines for experiments, and the advantages and disadvantages of each model are compared from the following 5 indicators:

(1) Mean Absolute Error (MAE), which can better reflect the actual situation of the predicted value error. The closer the MAE value is to 0 , the smaller is the error.

$$
M A E=\frac{1}{m} \sum_{i=1}^{m}\left|y_{i}-\hat{y}_{i}\right|
$$

(2) Root Mean Square Error (RMSE), the mean value of the square root of the error between the predicted value and the true value. The closer the RMSE value is to 0 , the more accurate the prediction result.

$$
R M S E=\sqrt{\frac{1}{m} \sum_{i=1}^{m}\left(y_{i}-\hat{y}_{i}\right)^{2}}
$$

(3) R-square $\left(R^{2}\right)$, is often used to measure the accuracy of the regression model, and the result is between $[0,1]$.

$$
R^{2}=\frac{S S R}{S S T}=\frac{\sum_{i=1}^{m} w_{i}\left(\hat{y}_{i}-\overline{\mathrm{y}}_{i}\right)^{2}}{\sum_{i=1}^{m} w_{i}\left(y_{i}-\overline{\mathrm{y}}_{i}\right)^{2}}=\frac{\sum_{i=1}^{m}\left(\hat{y}_{i}-\overline{\mathrm{y}}_{i}\right)^{2}}{\sum_{i=1}^{m}\left(y_{i}-\overline{\mathrm{y}}_{i}\right)^{2}}
$$

(4) The number of models, which can more intuitively reflect the complexity of the model.

(5) Time consuming of prediction. This comparison experiment will run on the Intel®Core ${ }^{\mathrm{TM}}$ I7-8700T CPU to 
measure the time taken for each saved the model to predict the target coordinates.

Table 2 shows the results of comparative experiments in this paper. Through comparative experiments, it is found that in terms of accuracy, the D-GRU positioning model proposed in this paper performs best on MAE, RMSE and R-square. Compared with the baseline algorithm DNN, SVR and GRNN: D-GRU reduces MAE by 0.348, 0.356, 0.129 respectively; reduce RMSE by $0.38,0.532,0.185$ respectively; and improve R-square by $0.057,0.099$ and 0.025 respectively. However, RNN performed the worst because it could not solve the long-term dependency problem. In terms of performance, SVR needs to establish regression models both in horizontal and vertical coordinates, so two models are needed. After GRNN determines the smoothing factor, it is an algorithm process and does not need to save the model. The rest of the algorithms only need to save one model. In terms of prediction time, SVR takes the shortest time, only $0.101 \mathrm{~s}$, because its prediction is only related to supporting vectors. However, GRNN takes the longest time, 3.896s, because GRNN needs to calculate the value of Guass function between the test set and all the training sets every time it predicts coordinates. The prediction time of the D-GRU positioning model proposed in this paper is only $0.371 \mathrm{~s}$, which meets the research needs.

Table 2. Analysis table of experimental results

\begin{tabular}{cccccccc}
\hline & D-GRU & GRU & LSTM & RNN & DNN & SVR & GRNN \\
\hline MAE & 0.302 & 0.335 & 0.359 & 1.240 & 0.650 & 0.658 & 0.431 \\
RMSE & 0.426 & 0.467 & 0.504 & 1.588 & 0.806 & 0.958 & 0.611 \\
R $^{2}$ & 0.975 & 0.970 & 0.966 & 0.663 & 0.918 & 0.876 & 0.950 \\
Models & 1 & 1 & 1 & 1 & 1 & 2 & 0 \\
TIME & $0.371 \mathrm{~s}$ & $0.228 \mathrm{~s}$ & $0.263 \mathrm{~s}$ & $0.124 \mathrm{~s}$ & $0.163 \mathrm{~s}$ & $0.101 \mathrm{~s}$ & $3.896 \mathrm{~s}$ \\
\hline
\end{tabular}

Table 3 shows the result of the comparation between D_GRU and three baselines (DNN, SVR and GRNN) on the 5 RFID training positions. And we can find that D_GRU performs better in most.

Table 3. Compare results with baselines

\begin{tabular}{cccccc}
\hline & $(6.2,0.8)$ & $(1.75,3.83)$ & $(7.68,1.81)$ & $(9.46,8.68)$ & $(4.74,3.28)$ \\
\hline D_GRU & $(6.3,0.74)$ & $(1.75,3.71)$ & $(8.05,1.29)$ & $(9.5,8.82)$ & $(4.95,2.94)$ \\
DNN & $(6.12,1.57)$ & $(2.13,3.5)$ & $(7.9,1.97)$ & $(8.45,8.07)$ & $(5.46,2.62)$ \\
SVR & $(6.9,1.15)$ & $(1.29,3.36)$ & $(8.08,2.62)$ & $(9.63,7.01)$ & $(4.8,2.75)$ \\
GRNN & $(7.15,1.83)$ & $(1.92,3.76)$ & $(8.07,1.9)$ & $(9.53,7.36)$ & $(4.82,3.25)$ \\
\hline
\end{tabular}

In addition, our experiment generated another random 20 sets of test data and used the stored D-GRU indoor positioning model that has been trained to forecast the position. The results are shown in Figure 7. The star represents the reallocation of the tag, while the circle represents the predicted location by the model. Among the prediction results of multiple points, the largest positioning error is about $0.6 \mathrm{~m}$, and most of the prediction errors are less than $0.4 \mathrm{~m}$. Experiment results show that the positioning model proposed in this paper can effectively perform indoor positioning. 


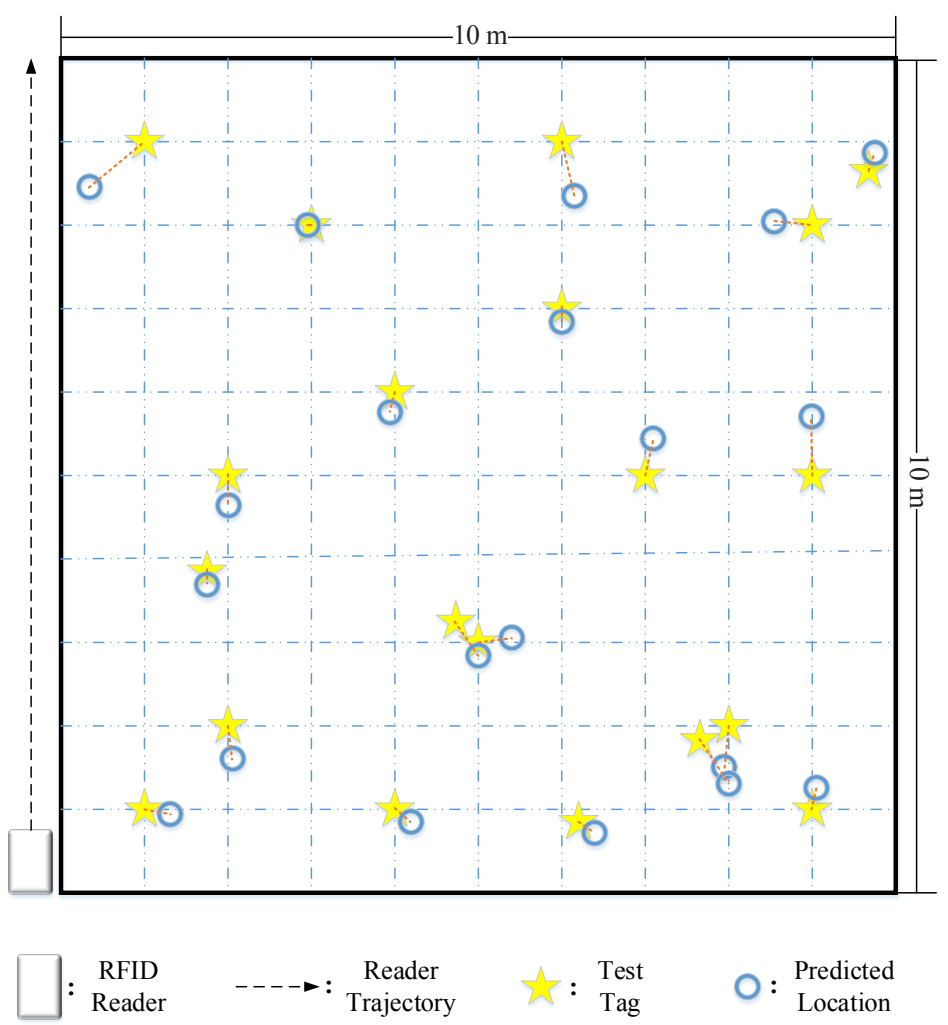

Figure 7. Result of positioning

\section{Discussion and conclusion}

By aiming at the cost and accuracy problems of contemporary indoor positioning technology, this paper has proposed a new Double-order GRU (D-GRU) based on single reader indoor positioning algorithm, which reduces the number of readers and improves positioning accuracy. On the simulation data set generated in this paper, D-GRU is higher than the baseline DNN, SVR and GRNN algorithms in MAE, RMSE and R-square indicators. Depending on its double order structure of the model, D-GRU also performs better than similar models like GRU, LSTM and RNN. However, this paper has used a mobile reader to replace multiple fixed-point readers, and its uneven motion is the main factor causing errors. In addition, we have simulated the impact of multipath effects by increasing noise in distance and signal strength, which may not be sufficient to simulate a complex indoor environment. Therefore, it is necessary to collect more data to locate in the indoor environment. In future research, we will conduct related research and work on these shortcomings.

\section{Acknowledgements}

Fujian province industrial field regional development project in 2019, intelligent mine construction and industrialization based on Internet of things and virtual reality, project number: 2019H4021.

Open fund of State Key Laboratory of Process Automation in Mining \& Metallurgy and Beijing Key Laboratory of Process Automation in Mining \& Metallurgy.

Project Name: Research on big data analysis method of concentration and metallurgy industry

Project number: BGRIMM-KZSKL-2019-03 


\section{References}

[1] Maung NAM, Zaw W. Comparative study of RSS-based indoor positioning techniques on two different WiFi frequency bands. 2020 17th International Conference on Electrical Engineering/Electronics, Computer, Telecommunications and Information Technology (ECTI-CON), Phuket, Thailand. 2020. p. 185-188. Available from: doi: 10.1109/ECTI-CON49241.2020.9158211.

[2] Xiao TT, Liao XY, Hu K, Yu M. Study of fingerprint location algorithm based on WiFi technology for indoor localization. 10th International Conference on Wireless Communications, Networking and Mobile Computing (WiCOM 2014), Beijing. 2014. p. 604-608. Available from: doi: 10.1049/ic.2014.0168.

[3] Rida ME, Liu F, Jadi Y, Algawhari AAA, Askourih A. Indoor location position based on bluetooth signal strength. 2015 2nd International Conference on Information Science and Control Engineering (ICISCE). 2015. p. 769-773.

[4] Montaser A, Moselhi O. RFID indoor location identification for construction projects. Automation in Construction. 2014; 39(1): 167-179.

[5] Yao W, Ma L. Research and application of indoor positioning method based on fixed infrared beacon. $201837^{\text {th }}$ Chinese Control Conference (CCC), Wuhan. 2018. p. 5375-5379. Available from: doi: 10.23919/ ChiCC.2018.8482658.

[6] Sosa-Sesma S, Perez-Navarro A. Fusion system based on WiFi and ultrasounds for in-home positioning systems: The UTOPIA experiment. 2016 International Conference on Indoor Positioning and Indoor Navigation (IPIN), Alcala de Henares. 2016. p. 1-8. Available from: doi: 10.1109/IPIN.2016.7743622.

[7] Fu J, Fu Y, Xu D. Application of an adaptive UKF in UWB indoor positioning. 2019 Chinese Automation Congress (CAC), Hangzhou, China. 2019. p. 544-549. Available from: doi: 10.1109/CAC48633.2019.8996692.

[8] Liu X, Wen M, Qin GH, Liu R. LANDMARC with improved k-nearest algorithm for RFID location system. 2016 2nd IEEE International Conference on Computer and Communications (ICCC), Chengdu. 2016. p. 2569-2572. Available from: doi: 10.1109/CompComm.2016.7925162.

[9] Zhao Y, Liu Y, Ni LM. VIRE: Active RFID-based localization using virtual reference elimination. 2007 International Conference on Parallel Processing (ICPP 2007), Xi'an. 2007. p. 56-56. Available from: doi: 10.1109/ ICPP.2007.84.

[10] Jin GY, Lu XY, Park MS. An indoor localization mechanism using active RFID tag. IEEE International Conference on Sensor Networks, Ubiquitous, and Trustworthy Computing (SUTC'06), Taichung. 2006. p. 4. Available from: doi: 10.1109/SUTC.2006.1636157.

[11] Wu L, Huang LY. Improvement of location methods based on RFID. The Journal of China Universities of Posts and Telecommunications. 2013; 20(6): 36-41.

[12] Zou Z, Deng T, Zou Q, Sarmiento MD, Jonsson F, Zheng L. Energy detection receiver with TOA estimation enabling positioning in passive UWB-RFID system. 2010 IEEE International Conference on Ultra-Wideband, Nanjing. 2010. p. 1-4. Available from: doi: 10.1109/ICUWB.2010.5615993.

[13] Deng Y, Zhu Y, Yang YF, Cheng XH, Li CQ. Research on RFID indoor location algorithm based on BP neural network. Small Microcomputer System. 2019; 40(8): 1707-1712.

[14] Zhao YL, An SB. Wi-Fi indoor localization algorithm based on kernel K-means and SVM classification regression. Information Technology. 2018; 1: 113-117.

[15] Song NJ, Cui YH. RFID indoor positioning algorithm based on genetic algorithm optimization GRNN. Computer Engineering. 2019; 45(11): 298-302, 308.

[16] Woo S, Byun J, Kim S, Nguyen HM, Im J, Kim D. RNN-Based personalized activity recognition in multi-person environment using RFID. 2016 IEEE International Conference on Computer and Information Technology (CIT), Nadi. 2016. p. 708-715. Available from: doi: 10.1109/CIT.2016.16.

[17] Christopher O. Understanding lstm networks. 2015. Available from: http://colah.github.io/posts/2015-08-Understanding-LSTMs. [Accessed 20th September 2020].

[18] Chung J, Gulcehre C, Cho KH, Bengio Y. Empirical evaluation of gated recurrent neural networks on sequence modeling. arXiv preprint. arXiv: 1412.3555. 2014. [Accessed 20th September 2020]. 